Check for updates

Cite this: Mater. Adv., 2021,

2, 2272

Received 2nd December 2020

Accepted 8th March 2021

DOI: 10.1039/d0ma00945h

rsc.li/materials-advances

\section{Formamide iodide: a new cation additive for inhibiting $\delta$-phase formation of formamidinium lead iodide perovskite $\uparrow$}

\author{
Itaru Raifuku, ${ }^{a}$ Yu-Hsien Chiang, ${ }^{a}$ Cheng-Hung Hou, (D) ${ }^{\mathrm{b}}$ Ming-Hsien Li, ${ }^{\text {ac }}$ \\ Chen-Fu Lin, ${ }^{a}$ Pei-Ying Lin, ${ }^{a}$ Jing-Jong Shyue ${ }^{(D)}{ }^{b}$ and Peter Chen (D) *ad
}

Perovskite solar cells (PSCs) employing organic-inorganic hybrid lead perovskite have attracted much attention as promising next generation solar cells because of their low fabrication cost and extremely high power conversion efficiency (PCE). Exploring new perovskite materials and additives is one of the effective strategies to improve the performance of PSCs. Here, we synthesized formamide iodide (FoAI) and applied it as both a cation material and additive. Although it was revealed that FoAl is not incorporated in the A-site of the perovskite structure, we found that the FoAl additive suppresses $\delta$-FAPbl ${ }_{3}$ formation and improved the performance of $\mathrm{FAPbl}_{3}$ based PSCs. The PCE was improved from $12.29 \%$ to $14.49 \%$ by adding 5 mol\% of FoAl in the precursor solution. Meanwhile, we found that FoAl additive can also improve the performance of triple-cation PSCs. We believe that FoAI is one of the promising additives to boost the PCE of PSCs without any influence on the composition of the perovskite materials.

\section{Introduction}

Perovskite solar cells (PSCs) employing organic-inorganic hybrid lead perovskite $\left(\mathrm{APbX}_{3}, \mathrm{~A}\right.$ : organic cation, $\mathrm{X}$ : halogen) have recently attracted much attention as promising next generation solar cells because of their low fabrication cost and extremely high power conversion efficiency (PCE). ${ }^{1-3}$ A PCE of over 25\% has been achieved because of excellent optoelectronic properties of the perovskite compounds such as strong absorption, low

\footnotetext{
${ }^{a}$ Department of Photonics, National Cheng Kung University, No. 1, University Rd, Tainan 70101, Taiwan. E-mail: petercyc@ncku.edu.tw

${ }^{b}$ Research Center for Applied Science, Academia Sinica, 128 Academia Rd, Sec. 2, Nankang, Taipei 115, Taiwan

'Department of Applied Materials and Optoelectronic Engineering, National Chi Nan University, No. 1, Daxue Rd, Nantou 54561, Taiwan

${ }^{d}$ Hierachical Green-Energy Materials (Hi-GEM) Research Center, National Cheng Kung University, No. 1, University Rd, Tainan 70101, Taiwan

† Electronic supplementary information (ESI) available. See DOI: 10.1039/ d0ma00945h
}

exciton binding energy and long carrier lifetime. ${ }^{4-7}$ Flexible PSCs can also be achieved owing to low process temperature. ${ }^{8,9}$ Moreover, PSCs can work effectively even under low illuminance conditions such as under indoor lighting; thus, various applications are expected..$^{10-13}$

Numerous strategies have been demonstrated to achieve high PCE PSCs such as solvent engineering, additive engineering, and interface modification. ${ }^{14-18}$ One of the effective strategies is modifying the composition of perovskite compounds. In the early stage of investigations, methylammonium lead iodide ( $\left.\mathrm{MAPbI}_{3}\right)$ was mainly used as the light absorber. A few years later, many researchers shifted to investigating mixed-cation perovskites such

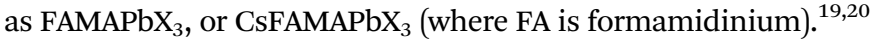
Mixed-cation perovskites have successfully shown better PCE and stability than conventional PSCs employing mono-cation perovskites. Currently, mono-cation $\mathrm{FAPbI}_{3}$ has been the focus of halide perovskites again owing to its optimal bandgap for single junction solar cells. In the early investigations of $\mathrm{FAPbI}_{3}$ PSCs, researchers suffered from poor efficiency and stability due to the formation of $\delta$-FAPbI 3 , which is a non-photoactive phase of $\mathrm{FAPbI}_{3} \cdot{ }^{21}$ Nowadays, phase pure $\alpha-\mathrm{FAPbI}_{3}$ films have been achieved by several strategies such as using pre-synthesized $\mathrm{FAPbI}_{3}$ powder as a precursor ${ }^{22}$ or adding additives in the precursor solution. ${ }^{23}$ To the best of our knowledge, the highest PCE of $25.17 \%$ was achieved with $\mathrm{FAPbI}_{3}$ employing a little amount of methylenediammonium dichloride and CsI as the additive. ${ }^{3}$

Cation also affects the structure of perovskite compounds. Small cations such as MA, FA, Cs and these mixtures form 3D structured perovskite. Meanwhile, large cations such as phenethylammonium or butylammonium form 2D structured perovskite. 2D structured perovskite compounds show quite different characteristics such as stability and optical properties from 3D structured ones. $^{24}$

As mentioned above, cation materials affect the characteristics of perovskite compounds. Therefore, exploring novel cation materials is an important work for further development of perovskite optoelectronics devices. Here, we have synthesized formamide 
iodide (FoAI) and investigated the potential of FoAI as a cation material for PSCs. It was revealed that FoAI itself has very limited inclusion in the $3 \mathrm{D}$ perovskite and does not change the perovskite structure from time-of-flight secondary ion mass spectrometry (ToF-SIMS), X-ray diffraction (XRD) patterns and Fourier transform infrared (FT-IR) spectroscopy. However, we found that FoAI suppresses the formation of $\delta$-FAPbI ${ }_{3}$ when it is applied as an additive for $\mathrm{FAPbI}_{3}$ perovskite. Moreover, it was revealed that FoAI improves the photovoltaic performance of PSCs for both pure $\mathrm{FAPbI}_{3}$ and mixed-cation perovskite compounds. Based on experimental results, we believe that FoAI is one of the promising additives to boost the PCE of PSCs without influence on the composition of perovskite materials.

\section{Experimental}

\section{Materials}

Methylammonium bromide and FAI were purchased from Dyesol. $\mathrm{PbI}_{2}, \mathrm{PbBr}_{2}$, and CsI were purchased from TCI. $\mathrm{SnO}_{2}$ colloidal dispersion liquid $\left(15 \%\right.$ in $\left.\mathrm{H}_{2} \mathrm{O}\right)$ was purchased from Alfa aesar. All other chemicals and solvents were received from Sigma Aldrich and used without purification.

\section{FoAI synthesis}

Formamide (Sigma, $\geq 99 \%$ ) was mixed with $2 x$ molar excess of $57 \% \mathrm{w} / \mathrm{w}$ hydroiodic acid and stirred at $50{ }^{\circ} \mathrm{C}$ for $10 \mathrm{~min}$. After drying at $100{ }^{\circ} \mathrm{C}$ overnight, a white-yellow powder was formed. The formed powder was washed with diethyl ether and recrystallized with ethanol at least five times and dried in an oven overnight.

\section{Device fabrication}

Glass substrates (10 $\mathrm{Ohm} \mathrm{sq}^{-1}$, Pilkington TEC10) coated with fluorine-doped tin oxide (FTO) were etched using zinc powder and $\mathrm{HCl}$ and then rinsed with pure water, ethanol, and acetone. To prepare the electron transport layer, titanium diisopropoxide bis(acetylacetonate) (75 wt\% in isopropanol, Sigma-Aldrich) diluted in ethanol $(1: 39$, volume ratio) was sprayed on the FTO-coated substrates at $475{ }^{\circ} \mathrm{C}$. Next, the substrates were annealed at $475{ }^{\circ} \mathrm{C}$ for $30 \mathrm{~min}$. After cooling the substrate to room temperature, $\mathrm{SnO}_{2}$ colloidal solution diluted in pure water $(1: 2$ weight ratio) was spincoated on the $\mathrm{TiO}_{2}$ layer at $5000 \mathrm{rpm}$ for $30 \mathrm{~s}$ and annealed at $150{ }^{\circ} \mathrm{C}$ for $30 \mathrm{~min}$. To form $\mathrm{FA}_{1-x} \mathrm{FoA}_{x} \mathrm{PbI}_{3}$ films, $1 \mathrm{M}$ precursor solution in a $8: 1$ volume ratio of DMF/DMSO solution was spincoated on the $\mathrm{SnO}_{2}$ layer and annealed at $170{ }^{\circ} \mathrm{C}$ for $10 \mathrm{~min}$. The precursor solution was prepared by mixing $1 \mathrm{M} \mathrm{FAPbI}_{3}$ solution and a solution including $1 \mathrm{M} \mathrm{PbI}_{2}$ and $1 \mathrm{M}$ FoAI with a preferred volume ratio. To form triple-cation perovskite films, $1.3 \mathrm{M} \mathrm{FA}_{0.81} \mathrm{MA}_{0.10} \mathrm{Cs}_{0.09} \mathrm{PbI}_{2.70} \mathrm{Br}_{0.30}$ solution in $7: 3$ volume ratio of DMF/DMSO solution was spin-coated on a $\mathrm{SnO}_{2}$ layer and annealed at $100{ }^{\circ} \mathrm{C}$ for $1 \mathrm{~h}$. The precursor solution was prepared by mixing $1.3 \mathrm{M} \mathrm{FA}_{0.9} \mathrm{Cs}_{0.1} \mathrm{PbI}_{3}$ solution and $1.3 \mathrm{M}$ $\mathrm{MAPbBr}_{3}$ solution (9:1, volume ratio). Both spin-coating processes were as follows: first, the substrates were accelerated to $2000 \mathrm{rpm}$ for $10 \mathrm{~s}\left(200 \mathrm{rpm} \mathrm{s}^{-1}\right)$ and then spun at $5000 \mathrm{rpm}$ for $20 \mathrm{~s}\left(2000 \mathrm{rpm} \mathrm{s}^{-1}\right)$, and second, chlorobenzene $(150 \mu \mathrm{L})$ was dropped onto the rotating substrate for $10 \mathrm{~s}$ before the end of the spin-coating process. In the case of utilizing FoAI as an additive, a moderate amount of FoAI was diluted in perovskite precursor solution and processed with the above protocol. To form a hole transport layer, a solution of $2,2^{\prime}, 7,7^{\prime}$-tetrakis $\left(N, N^{\prime}\right.$-dip-methoxyphenylamine)-9,9'-spirobifluorene (17 mg) with 4-tertbutylpyridine $(6.9 \mu \mathrm{L})$ and lithium bis(trifluoromethanesulfonyl)imide $\left(4.2 \mu \mathrm{L}, 520 \mathrm{mg} \mathrm{mL}{ }^{-1}\right.$ in acetonitrile) in chlorobenzene $(240 \mu \mathrm{L})$ was spin-coated on the perovskite layer at $4000 \mathrm{rpm}$ for $30 \mathrm{~s}$ to deposit a hole transport layer (HTL). Finally, an Ag electrode was deposited by thermal evaporation on the hole transport layer.

\section{Characterization}

The scanning electron microscope (SEM) (SU8000, Hitachi) was performed to examine the top-view film morphology. The optical property was measured by a UV-vis spectrometer (U-4100, Hitachi). The $J-V$ characteristic measurements were conducted under AM 1.5G illumination $\left(100 \mathrm{~mW} \mathrm{~cm}^{-2}\right)$ using a solar simulation system (SS-F5-3A, Enlitech) and the device was connected to a source meter (Keithley 2401) for tracing the $J-V$ data. The light intensity was calibrated by certified standard silicon solar cells (SRC-2020-

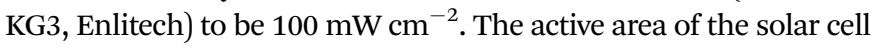
was masked by $0.15 \mathrm{~cm}^{2}$. A $300 \mathrm{~W}$ intensity xenon lamp (Newport), monochromatic (Newport Cornerstone 260), and source meter (Keithley 2401) were integrated to measure the device IPCE response. The XRD measurements were measured with a D8 ADVANCE ECO (Bruker). The photoluminescence (PL) measurements (ProtrustTech MRI) were carried out by using a diode laser with a $532 \mathrm{~nm}$ light source (intensity: $127 \mathrm{~mW}$ ). The FT-IR spectra were measured with a VERTEX 70 (Bruker). The band position of the perovskite films was evaluated with a Kelvin probe system and ambient pressure photoemission spectroscopy (APS) system (KP Technology Ltd.). ToF-SIMS was performed with PHI TRIFT $\mathrm{V}$ nanoTOF (ULVAC-PHI) at Academis Sinica. Details of the ToF-SIMS measurement for perovskite materials are described elsewhere. $^{25}$

\section{Results and discussion}

The molecular structure of FoAI is shown in Fig. 1(a). FoAI has a similar structure to FAI, which is a well-known cation shown in Fig. 1(b). The powder XRD patterns of both compounds are shown in Fig. S1 (ESI $\dagger$ ). Fig. 1(c) shows the FT-IR spectra of FoAI and FAI. A characteristic $\mathrm{C}=\mathrm{N}$ stretching peak was observed in (a)<smiles>NC=O</smiles>

(b)

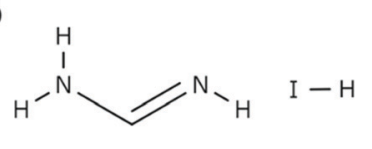

(c)

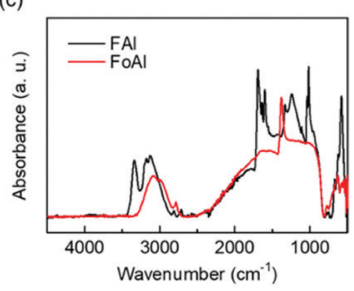

Fig. 1 Molecular structure of (a) formamide iodide (FoAl) and (b) formamidinium iodide (FAI). (c) FT-IR spectra of FoAl and FAI. 
the spectrum of FAI at around $1700 \mathrm{~cm}^{-1}$. On the other hand, the $\mathrm{C}=\mathrm{O}$ stretching bond from amide compounds, which usually appears around $1650-1515 \mathrm{~cm}^{-1}$ was not observed in the spectrum of FoAI. It is known that formamide has several resonance structures as shown in Fig. S2 (ESI $\dagger){ }^{26}$ Therefore, there is a possibility that the state which has $\mathrm{C}=\mathrm{O}$ bonding is unstable and the signal from $\mathrm{C}=\mathrm{O}$ became weak. We are assuming that the signal of FoAI at around $1400 \mathrm{~cm}^{-1}$ is $\mathrm{C}-\mathrm{N}$ stretching or $\mathrm{C}-\mathrm{OH}$ bending. The position of the FT-IR signals and related bonding are summarized in Tables S1 and S2 (ESI $\dagger$ ). Fig. S3 (ESI $\dagger$ ) shows an XRD pattern of a film obtained by spin-coating a solution including $\mathrm{PbBr}_{2}$ and FoAI with a molar ratio of $1: 1$. The resulting film showed XRD patterns of $\mathrm{PbI}_{2}$ and $\mathrm{PbBr}_{2}$. This result indicates that FoAI powder includes iodide in the structure. Also, a diffraction peak that cannot be assigned to $\mathrm{PbI}_{2}$ or $\mathrm{PbBr}_{2}$ film was observed at around $12^{\circ}$ in the XRD patterns of FoAI- $\mathrm{PbBr}_{2}$ mixed film. It is reported that $\mathrm{PbBr}_{2}$ also has a diffraction peak at around $12^{\circ} .{ }^{27}$ Therefore, it is considered that the possible origin of the peak is from an intermediate mixed halide compound between $\mathrm{PbI}_{2}$ and $\mathrm{PbBr}_{2}$ such as $\operatorname{PbI}_{x} \mathrm{Br}_{(2-x)}$. Actually, it is calculated that $\mathrm{PbIBr}$ shows a diffraction pattern around $12^{\circ} .^{28}$ This compound is also considered as one of the possible origins of the diffraction peak.

Then, we investigated whether FoA could be inserted in the A-site of perovskite compounds with a system of $\mathrm{FA}_{1-x} \mathrm{FoA}_{x} \mathrm{PbI}_{3}$. Fig. 2(a) shows photographs of $\mathrm{FA}_{1-x} \mathrm{FoA}_{x} \mathrm{PbI}_{3}$ films prepared under various stoichiometries. The $x=0, \mathrm{FAPbI}_{3}$ film showed black color and it was confirmed that the film mainly consists of $\alpha-\mathrm{FAPbI}_{3}$ with the presence of $\delta$ phase from XRD patterns as shown in Fig. 2(b). By contrast, the $x=1$, FoAPbI $_{3}$ film showed bright yellow color which is similar to $\mathrm{PbI}_{2}$ films. The XRD patterns of $\mathrm{FoAPbI}_{3}$ films revealed that the film is mainly composed of $\mathrm{PbI}_{2}$. FT-IR spectroscopy was carried out to confirm whether FoA is present in the resulting films or not. Fig. 2(c) shows FT-IR spectra of each composition film. Films containing less FoA showed clear signals arising from $\mathrm{N}-\mathrm{H}$ stretching and $\mathrm{C}=\mathrm{N}$ stretching in the spectra. By contrast, films containing a large amount of FoA did not show any signals in their spectra. Results from XRD and FT-IR measurements indicate that FoA does not form perovskite.

(a)
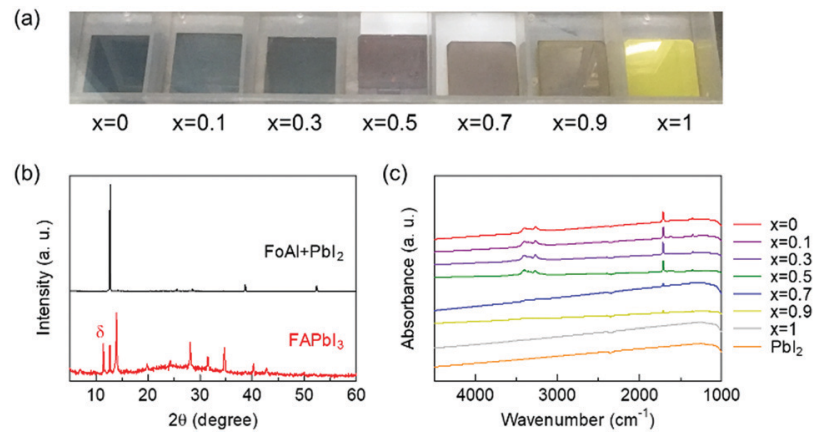

Fig. 2 (a) Photographs of $\mathrm{FA}_{1-x} \mathrm{FoA}_{x} \mathrm{~Pb}_{3}$ films, where $\mathrm{FA}$ and FoA are formamidinium and formamide, respectively. (b) XRD patterns of $x=0\left(\mathrm{FAPbl}_{3}\right)$ and $x=1\left(\mathrm{FoAPbl}_{3}\right)$ films. (c) $\mathrm{FT}$-IR spectra of $\mathrm{FA}_{1-x} \mathrm{FoA}_{x} \mathrm{Pbl}{ }_{3}$ films.
We found that a solution including FoAI and $\mathrm{PbI}_{2}$ does not form a precipitate even if chlorobenzene, which is an antisolvent, is added in the solution. On the other hand, the pure $\mathrm{PbI}_{2}$ solution immediately forms a precipitate as shown in Fig. S4 (ESI $\dagger$ ). This phenomenon indicates that there is a possibility that FoAI forms an adduct or complex with $\mathrm{PbI}_{2}$ and thus, improves the solubility of $\mathrm{PbI}_{2}$. Then, we applied FoAI as an additive for PSCs employing $\mathrm{FAPbI}_{3}$ and mixed-cation perovskite as the light absorber.

Fig. 3(a) shows $J-V$ curves of PSCs employing $\mathrm{FAPbI}_{3}$ with and without FoAI as an additive in the precursor solution. The FoAI added sample showed higher PCE particularly with improved short-circuit current density $\left(J_{\mathrm{SC}}\right)$ compared to the control devices. Fig. 3(b) and (c) show external quantum efficiency (EQE) spectra of PSCs and absorbance spectra of perovskite films, respectively. The FoAI added device showed an entirely higher EQE value than the control device even though there is almost no change in the absorbance spectra of perovskite films. The band positions of $\mathrm{FAPbI}_{3}$ films with and without FoAI additive were evaluated with the Kelvin probe method and APS measurements. Fig. S5(a) (ESI $\dagger$ ) shows the difference of the surface potential of both films against a reference tip. Fig. S5(b) (ESI $\dagger$ ) shows photoemission spectra of both films obtained by APS measurement. There were no obvious changes in surface potential and photoemission spectra, indicating that both perovskite films have same work function and ionization potential. Also, both films showed the same absorption edge as shown in Fig. 3(c). From these results, we assume that the FoAI additive does not affect the band position of $\mathrm{FAPbI}_{3}$ films. Fig. 3(d) shows the XRD patterns of the $\mathrm{FAPbI}_{3}$ films with and without FoAI additive. The $\mathrm{FAPbI}_{3}$ film which does not include FoAI additive showed a diffraction peak originated from $\delta-\mathrm{FAPbI}_{3}$, which is not a (a)

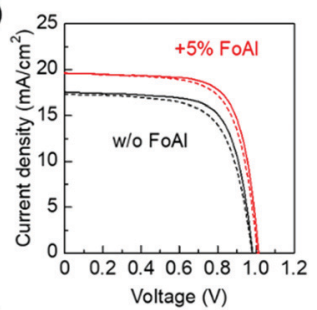

(c)

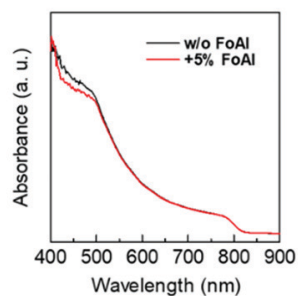

(b)

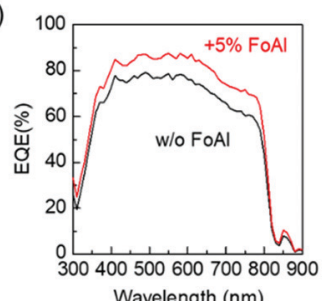

(d)

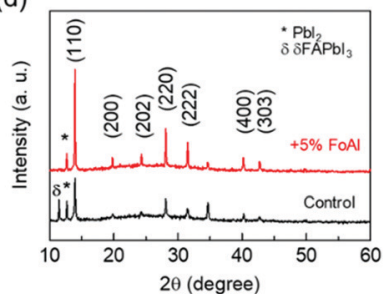

Fig. 3 (a) $J-V$ curves of $\mathrm{FAPbl}_{3}$ perovskite solar cells with and without FoAl additive. The dashed and solid lines represent $J-V$ curves obtained at forward ( $J_{S C}$ to $V_{O C}$ ) and reverse $\left(V_{O C}\right.$ to $\left.J_{S C}\right)$ scan, respectively. (b) External quantum efficiency (EQE) curves of $\mathrm{FAPbl}_{3}$ perovskite solar cells with and without FoAl additive. (c) Absorbance spectra of $\mathrm{FAPbl}_{3}$ perovskite films with and without FoAl additive. (d) XRD patterns of $\mathrm{FAPbl}_{3}$ perovskite films with and without FoAl additive. The peaks marked with * and $\delta$ are originated from $\mathrm{Pbl}_{2}$ and $\delta-\mathrm{FAPb}_{3}$, respectively. 
photoactive phase for solar cells. On the other hand, FoAI added films did not show diffraction peaks from $\delta$-FAPbI ${ }_{3}$. This result indicates that the FoAI additive suppresses the formation of $\delta-\mathrm{FAPbI}_{3}$.

Fig. S6 (ESI $\dagger$ ) shows PL spectra of pure $\mathrm{FAPbI}_{3}$ and FoAI added $\mathrm{FAPbI}_{3}$ films. Both films show a PL peak at around $800 \mathrm{~nm}$, which is consistent with the absorption edge of the films. Fig. S7 (ESI $\dagger$ ) shows SEM images of pure $\mathrm{FAPbI}_{3}$ and FoAI added $\mathrm{FAPbI}_{3}$ films. There was almost no change in the morphology of $\mathrm{FAPbI}_{3}$ films by adding FoAI.

Fig. S8 (ESI $\dagger$ ) shows dark $I-V$ curves of electron only devices employing pure and FoAI added $\mathrm{FAPbI}_{3}$ films. The device structure is $\mathrm{FTO} / \mathrm{TiO}_{2} / \mathrm{SnO}_{2} /$ perovskite/PCBM/Ag. The current versus bias behavior can be divided into three different regions, ohmic region, trap filling region, and space charge limited current region. The voltage where the behavior changes from ohmic to trap filling is called the trap-filled limit voltage $\left(V_{\mathrm{TFL}}\right)$. The relationship between $V_{\text {TFL }}$ and trap density $\left(N_{\mathrm{t}}\right)$ can be described as follows; $N_{\mathrm{t}}=2 \varepsilon_{0} \varepsilon V_{\mathrm{TFL}} / e L^{2}$, where $\varepsilon_{0}$ is the vacuum permittivity, $\varepsilon$ is the relative dielectric constant of perovskite film, $e$ is the electron charge, and $L$ is the film thickness. ${ }^{29}$ Comparing the dark $I-V$ curves, we found that the FoAI added sample showed slightly lower $V_{\text {TFL }}$ indicating that FoAI added films have less defects than pure $\mathrm{FAPbI}_{3}$ films.

Hysteresis index, defined as $\left(\mathrm{PCE}_{\text {reverse }}-\mathrm{PCE}_{\text {forward }}\right) / \mathrm{PCE}_{\text {reverse, }}$, of $\mathrm{FAPbI}_{3}$ PSCs was calculated and summarized in Table S3 $(\mathrm{ESI} \dagger) .{ }^{30}$ There was no significant difference in hysteresis index of $\mathrm{FAPbI}_{3}$ PSCs with and without FoAI additive.

We have also investigated the effect of FoAI on the stability of $\mathrm{FAPbI}_{3}$ PSCs. Fig. S9 (ESI $\dagger$ ) shows the normalized PCE of $\mathrm{FAPbI}_{3} \mathrm{PSCs}_{\mathrm{Nept}}$ at a maximum power point under continuous 1 sun irradiation without encapsulation. After 90 min irradiation, the PCE of pure $\mathrm{FAPbI}_{3}$ PSCs decreased to $42.7 \%$ of the initial value. By contrast, FoAI added PSCs kept a slightly higher value of $49.3 \%$. This result indicates that FoAI might also improve the stability of $\mathrm{FAPbI}_{3}$ PSCs under working conditions.

The $J-V$ characteristics of PSCs are summarized in Table 1. The PCE of our control sample (12.29\%) seems to be low considering the record efficiency $(>25 \%)$ of PSCs. However, it is comparable to the baseline of pure $\mathrm{FAPbI}_{3}$ devices whose PCE is around $13-17 \% .^{31-33}$ We believe that our method offers an alternative strategy to stabilize and improve the performance of pure $\mathrm{FAPbI}_{3}$ based devices.

Then, we applied FoAI additive in multi-cation PSCs, which are the baseline of high performing perovskite cells in general. (a)

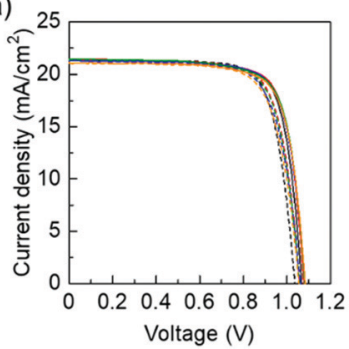

(b)

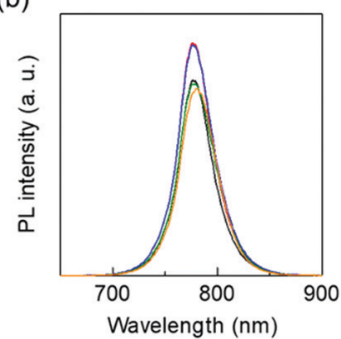

Fig. 4 (a) J-V curves of FoAl added triple-cation perovskite solar cells. (b) Photoluminescence $(\mathrm{PL})$ spectra of triple-cation perovskite films with and without FoAl additives. The black, red, blue, green, and orange lines represent $0,0.625,1.25,2.5$ and 5 mol\% FoAl added samples, respectively.

Fig. 4(a) shows $J-V$ curves of triple-cation PSCs with and without FoAI additives. EQE spectra of each sample are shown in Fig. S10 (ESI $\dagger$ ). The $V_{\text {OC }}$ was slightly improved by adding FoAI in the precursor solution. Fig. 4(b) shows PL spectra of triple-cation perovskite films with and without FoAI additives. Perovskite films containing 0.625 and 1.25 mol\% of FoAI showed stronger PL intensity than the control films, indicating that FoAI helps the formation of less defect perovskite films and resulted in improved $V_{\text {OC }}$. There were no obvious changes in absorbance spectra, XRD patterns, and morphology as shown in Fig. S11 and S12 (ESI $\dagger)$. Dark $I-V$ characteristics of the electron only device employing triple-cation perovskite films are shown in Fig. $\mathrm{S} 13$ (ESI $\dagger$ ). In contrast to $\mathrm{FAPbI}_{3}$ films, there was no significant difference in $V_{\text {TFL }}$ of both triple-cation perovskite films. The $J-V$ characteristics of triple-cation PSCs with and without FoAI additives are summarized in Table 2 . The hysteresis index of triple-cation PSCs was calculated and summarized in Table S4 (ESI $\dagger$ ). There was no significant difference in hysteresis index by FoAI concentration.

Fig. S14 (ESI $\dagger$ ) shows FT-IR spectra of FoAI added triplecation perovskite films. Although signals originated from $\mathrm{N}-\mathrm{H}$, $\mathrm{C}-\mathrm{N}$, and $\mathrm{C}=\mathrm{N}$ bonding of MAI and FAI were observed, there was no clear signal at around $1400 \mathrm{~cm}^{-1}$, where characteristic peaks were observed in the spectrum of FoAI (Fig. 1). This result also indicates that FoA is not included as an A-site cation of a perovskite structure.

We further investigated whether FoAI is included in the final perovskite films with ToF-SIMS which has a higher detection limit than FT-IR measurements. Fig. S15 (ESI $\dagger$ ) shows the ToFSIMS profile (positive ion) of pure $\mathrm{FAPbI}_{3}$ and FoAI added films.

Table $1 \mathrm{~J}-\mathrm{V}$ characteristics of $\mathrm{FAPb}_{3}$ perovskite solar cells. Values in brackets are from the best performing device under each condition

\begin{tabular}{|c|c|c|c|c|}
\hline & $J_{\mathrm{SC}}\left(\mathrm{mA} \mathrm{cm}^{-2}\right)$ & $V_{\mathrm{OC}}(\mathrm{V})$ & FF & PCE (\%) \\
\hline Control & $16.86 \pm 0.53$ & $0.964 \pm 0.023$ & $0.649 \pm 0.036$ & $10.71 \pm 1.10$ \\
\hline $\begin{array}{l}\text { (Forwara) } \\
\text { Control }\end{array}$ & $17.17+0.48$ & $0.977+0.007$ & $0.687+0.016$ & $11.67+0.63$ \\
\hline (Reverse) & $(17.55)$ & $(0.983)$ & $(0.704)$ & $(12.29)$ \\
\hline With FoAI & $18.98 \pm 0.92$ & $1.000 \pm 0.005$ & $0.685 \pm 0.010$ & $13.16 \pm 0.84$ \\
\hline (Forward) & (19.63) & $(1.007)$ & $(0.696)$ & (13.93) \\
\hline With FoAI & $19.05 \pm 0.75$ & $1.005 \pm 0.008$ & $0.717 \pm 0.010$ & $13.90 \pm 0.54$ \\
\hline (Reverse) & (19.59) & $(1.014)$ & $(0.721)$ & (14.49) \\
\hline
\end{tabular}


Table $2 \mathrm{~J}-V$ characteristics of triple-cation perovskite solar cells obtained by reverse scan ( $V_{\text {OC }}$ to $\left.J_{S C}\right)$

\begin{tabular}{lllll}
\hline FoAI $(\mathrm{mol} \%)$ & $J_{\text {SC }}\left(\mathrm{mA} \mathrm{cm}^{-2}\right)$ & $V_{\mathrm{OC}}(\mathrm{V})$ & FF & PCE $(\%)$ \\
\hline 0 & $21.30 \pm 0.17$ & $1.065 \pm 0.004$ & $0.765 \pm 0.008$ & $17.36 \pm 0.22$ \\
0.625 & $21.28 \pm 0.09$ & $1.077 \pm 0.006$ & $0.767 \pm 0.005$ & $17.58 \pm 0.10$ \\
1.25 & $21.28 \pm 0.02$ & $1.082 \pm 0.006$ & $0.757 \pm 0.005$ & $17.41 \pm 0.12$ \\
2.5 & $21.30 \pm 0.08$ & $1.077 \pm 0.003$ & $0.754 \pm 0.005$ & $17.30 \pm 0.18$ \\
5 & $21.25 \pm 0.16$ & $1.073 \pm 0.010$ & $0.756 \pm 0.006$ & $17.24 \pm 0.17$
\end{tabular}

It is difficult to conclude whether the FoA ion is included in the final films from positive ion mode because the FA ion and FoA ion have a similar $m / z$ value (FA: $m / z=45$, FoA: $m / z=46$ ). Actually, the pure $\mathrm{FAPbI}_{3}$ film showed a signal of $\mathrm{m} / \mathrm{z}=46$, which might result from isotopic atoms contained in FA ions. Then, we checked negative ions included in FoAI added $\mathrm{FAPbI}_{3}$ films. Fig. S16 (ESI $\dagger$ ) shows I and $\mathrm{O}$ ion distribution in FoAI added films. $\mathrm{O}$ ions were not detected in the perovskite films, indicating that FoAI is not included in the final films. We also could not detect the $\mathrm{O}$ ion in FoAI added triple cation perovskite films as shown in Fig. S17 (ESI $\dagger$ ).

From inhibited $\delta$-FAPbI ${ }_{3}$ formation (Fig. 3(d)) and enhanced PL intensity of perovskite films (Fig. 4(b)), there is a possibility that FoAI affects the crystal growth of perovskite films. It is also observed in the XRD patterns of the perovskite films (Fig. 3(d) and Fig. S11(b), ESI $\dagger$ ) that the FoAI added films showed stronger intensity than the pure perovskite films. This result also indicates that FoAI affects the crystal growth of perovskite films. One of the possible mechanisms is that FoAI forms adduct with $\mathrm{PbI}_{2}$ as mentioned above. It is known that adduct formation leads to highly efficient PSCs. ${ }^{34,35}$ Furthermore, it is reported that idodide defects in perovskite films affect the device performance of PSCs. ${ }^{36}$ There is a possibility that iodide from FoAI reduces the defects and improves the efficiency of PSCs. Although further investigation is necessary to clarify the detailed role of FoAI, we believe that FoAI is one of the promising materials to boost the PCE of PSCs.

\section{Conclusions}

In summary, we synthesized FoAI and applied it as a material for PSCs. Through the fabrication and evaluation of $\mathrm{FA}_{1-x} \mathrm{FoA}_{x} \mathrm{PbI}_{3}$ films, it was confirmed that FoAI is not incorporated in the perovskite structure. However, it was confirmed that FoAI improves the solubility of $\mathrm{PbI}_{2}$ probably due to the formation of an adduct or complex. When FoAI was applied as an additive for $\mathrm{FAPbI}_{3}$ based solar cells, it was confirmed that $\delta$-FAPbI formation was suppressed and the photovoltaic performance of $\mathrm{FAPbI}_{3}$ PSCs was improved. The PCE of $\mathrm{FAPbI}_{3}$ PSCs was improved from $12.29 \%$ to $14.49 \%$ by adding $5 \mathrm{~mol} \%$ of FoAI in the precursor solution. We found that FoAI additive also improves the photovoltaic performance of triple-cation (FA, MA, and Cs mixed-cation) PSCs. FoAI added triple-cation perovskite films showed higher PL intensity than control films and FoAI added PSCs showed slightly improved $V_{\text {OC }}$. From the above results, we assume that FoAI affects the crystal growth of perovskite films.
We believe that FoAI will be one of the promising materials to boost the performance of PSCs.

\section{Conflicts of interest}

There are no conflicts to declare.

\section{Acknowledgements}

We would like to thank the financial support from the Ministry of Science and Technology of Taiwan (MOST 107-2221-E-006190-MY3, MOST 108-3116-F-006-001, and 108-2218-E-006-043MY3). This work was financially supported by the Hierarchical Green-Energy Materials (Hi-GEM) Research Center, from The Featured Areas Research Center Program within the framework of the Higher Education Sprout Project by the Ministry of Education (MOE) and the Ministry of Science and Technology (MOST 109-2634-F-006-020) in Taiwan. This research was supported in part by the Higher Education Sprout Project, Ministry of Education to the Headquarters of University Advancement at the National Cheng Kung University (NCKU).

\section{References}

1 A. Kojima, K. Teshima, Y. Shirai and T. Miyasaka, J. Am. Chem. Soc., 2009, 131, 6050-6051.

2 M. Cai, Y. Wu, H. Chen, X. Yang, Y. Qiang and L. Han, Adv. Sci., 2017, 4, 1600269.

3 G. Kim, H. Min, K. S. Lee, D. Y. Lee, S. M. Yoon and S. I. Seok, Science, 2020, 370, 108.

4 M. Green, E. Dunlop, J. Hohl-Ebinger, M. Yoshita, N. Kopidakis and X. Hao, Prog. Photovolt., 2021, 29, 3-15.

5 G. Xing, N. Mathews, S. Sun, S. S. Lim, Y. M. Lam, M. Grätzel, S. Mhaisalkar and T. C. Sum, Science, 2013, 342, 344.

6 S. D. Stranks, G. E. Eperon, G. Grancini, C. Menelaou, M. J. P. Alcocer, T. Leijtens, L. M. Herz, A. Petrozza and H. J. Snaith, Science, 2013, 342, 341.

7 A. Miyata, A. Mitioglu, P. Plochocka, O. Portugall, J. T.-W. Wang, S. D. Stranks, H. J. Snaith and R. J. Nicholas, Nat. Phys., 2015, 11, 582-587.

8 S. Kang, J. Jeong, S. Cho, Y. J. Yoon, S. Park, S. Lim, J. Y. Kim and H. Ko, J. Mater. Chem. A, 2019, 7, 1107-1114.

9 D. Yang, R. Yang, S. Priya and S. Liu, Angew. Chem., Int. Ed., 2019, 58, 4466-4483.

10 I. Raifuku, Y. Ishikawa, S. Ito and Y. Uraoka, J. Mater. Chem. C, 2016, 120, 18986-18990.

11 M. Li, C. Zhao, Z.-K. Wang, C.-C. Zhang, H. K. H. Lee, A. Pockett, J. Barbé, W. C. Tsoi, Y.-G. Yang, M. J. Carnie, X.-Y. Gao, W.-X. Yang, J. R. Durrant, L.-S. Liao and S. M. Jain, Adv. Energy Mater., 2018, 8, 1801509.

12 R. Cheng, C.-C. Chung, H. Zhang, F. Liu, W.-T. Wang, Z. Zhou, S. Wang, A. B. Djurišić and S.-P. Feng, Adv. Energy Mater., 2019, 9, 1901980.

13 I. Raifuku, Y. Ishikawa, Y.-H. Chiang, P.-Y. Lin, M.-H. Li, Y. Uraoka and P. Chen, RSC Adv., 2019, 9, 32833-32838. 
14 N. J. Jeon, J. H. Noh, Y. C. Kim, W. S. Yang, S. Ryu and S. I. Seok, Nat. Mater., 2014, 13, 897-903.

15 Y. Zhou, M. Yang, W. Wu, A. L. Vasiliev, K. Zhu and N. P. Padture, J. Mater. Chem. A, 2015, 3, 8178-8184.

16 T. Li, Y. Pan, Z. Wang, Y. Xia, Y. Chen and W. Huang, J. Mater. Chem. A, 2017, 5, 12602-12652.

17 H. Zhou, Q. Chen, G. Li, S. Luo, T.-B. Song, H.-S. Duan, Z. Hong, J. You, Y. Liu and Y. Yang, Science, 2014, 345, 542.

18 Q. Jiang, Y. Zhao, X. Zhang, X. Yang, Y. Chen, Z. Chu, Q. Ye, X. Li, Z. Yin and J. You, Nat. Photonics, 2019, 13, 460-466.

19 W. S. Yang, J. H. Noh, N. J. Jeon, Y. C. Kim, S. Ryu, J. Seo and S. I. Seok, Science, 2015, 348, 1234.

20 M. Saliba, T. Matsui, J.-Y. Seo, K. Domanski, J.-P. CorreaBaena, M. K. Nazeeruddin, S. M. Zakeeruddin, W. Tress, A. Abate, A. Hagfeldt and M. Grätzel, Energy Environ. Sci., 2016, 9, 1989-1997.

21 S. Pang, H. Hu, J. Zhang, S. Lv, Y. Yu, F. Wei, T. Qin, H. Xu, Z. Liu and G. Cui, Chem. Mater., 2014, 26, 1485-1491.

22 Y. Zhang, S. Seo, S. Y. Lim, Y. Kim, S.-G. Kim, D.-K. Lee, S.H. Lee, H. Shin, H. Cheong and N.-G. Park, ACS Energy Lett., 2020, 5, 360-366.

23 Y. Liu, S. Akin, A. Hinderhofer, F. T. Eickemeyer, H. Zhu, J.-Y. Seo, J. Zhang, F. Schreiber, H. Zhang, S. M. Zakeeruddin, A. Hagfeldt, M. I. Dar and M. Grätzel, Angew. Chem., Int. Ed., 2020, 59, 15688-15694.

24 Y. Zheng, T. Niu, X. Ran, J. Qiu, B. Li, Y. Xia, Y. Chen and W. Huang, J. Mater. Chem. A, 2019, 7, 13860-13872.
25 C.-H. Hou, S.-H. Hung, L.-J. Jhang, K.-J. Chou, Y.-K. Hu, P.-T. Chou, W.-F. Su, F.-Y. Tsai, J. Shieh and J.-J. Shyue, ACS Appl. Mater. Interfaces, 2020, 12, 22730-22740.

26 C. R. Kemnitz and M. J. Loewen, J. Am. Chem. Soc., 2007, 129, 2521-2528.

27 Z. Ahmad and A. Mishra, J. Mater. Sci.: Mater. Electron., 2020, 31, 4672-4676.

28 K. Persson, The Materials Project, 2020, DOI: 10.17188/ 1276273.

29 D. Yang, R. Yang, K. Wang, C. Wu, X. Zhu, J. Feng, X. Ren, G. Fang, S. Priya and S. Liu, Nat. Commun., 2018, 9, 3239.

30 S. N. Habisreutinger, N. K. Noel and H. J. Snaith, ACS Energy Lett., 2018, 3, 2472-2476.

31 J.-W. Lee, Z. Dai, T.-H. Han, C. Choi, S.-Y. Chang, S.-J. Lee, N. De Marco, H. Zhao, P. Sun, Y. Huang and Y. Yang, Nat. Commun., 2018, 9, 3021.

32 F. Xie, C.-C. Chen, Y. Wu, X. Li, M. Cai, X. Liu, X. Yang and L. Han, Energy Environ. Sci., 2017, 10, 1942-1949.

33 S. Akin, E. Akman and S. Sonmezoglu, Adv. Funct. Mater., 2020, 30, 2002964.

34 N. Ahn, D.-Y. Son, I.-H. Jang, S. M. Kang, M. Choi and N.-G. Park, J. Am. Chem. Soc., 2015, 137, 8696-8699.

35 J.-W. Lee, H.-S. Kim and N.-G. Park, Acc. Chem. Res., 2016, 49, 311-319.

36 W. S. Yang, B.-W. Park, E. H. Jung, N. J. Jeon, Y. C. Kim, D. U. Lee, S. S. Shin, J. Seo, E. K. Kim, J. H. Noh and S. I. Seok, Science, 2017, 356, 1376. 